\title{
REVIEW
}

\section{Diagnosis and treatment of deep-vein thrombosis}

\author{
Dimitrios Scarvelis, Philip S. Wells
}

\section{ABSTRACT}

Deep-vein thrombosis (DVT) is a common condition that can lead to complications such as postphlebitic syndrome, pulmonary embolism and death. The approach to the diagnosis of DVT has evolved over the years. Currently an algorithm strategy combining pretest probability, D-dimer testing and compression ultrasound imaging allows for safe and convenient investigation of suspected lower-extremity thrombosis. Patients with low pretest probability and a negative D-dimer test result can have proximal DVT excluded without the need for diagnostic imaging. The mainstay of treatment of DVT is anticoagulation therapy, whereas interventions such as thrombolysis and placement of inferior vena cava filters are reserved for special situations. The use of low-molecular-weight heparin allows for outpatient management of most patients with DVT. The duration of anticoagulation therapy depends on whether the primary event was idiopathic or secondary to a transient risk factor. More research is required to optimally define the factors that predict an increased risk of recurrent DVT to determine which patients can benefit from extended anticoagulant therapy.

CMAJ 2006;175(9):1087-92

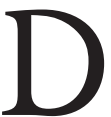
eep-vein thrombosis (DVT) has an estimated annual incidence of 67 per 100 ooo among the general populations. ${ }^{1,2}$ Despite adequate therapy, $\mathrm{I} \%$ to $8 \%$ of patients in whom pulmonary embolism develops will die, ${ }^{3-5}$ whereas others will experience long-term complications such as postphlebitic syndrome $(40 \%)^{6}$ and chronic thromboembolic pulmonary hypertension (4\%). Although anticoagulant therapy decreases the risk of recurrent thrombosis, the treatment also increases the risk of major hemorrhage. Before 1995 the approach was to image all patients with suspected DVT and to repeat tests I week later if results were negative. ${ }^{8,9}$ This approach was inefficient, since only 10\%-25\% of patients with suspected DVT were found to actually have the disorder and results of serial tests were usually negative. ${ }^{9-12}$ Over the last Io years new strategies for diagnosing and treating suspected DVT have been introduced.

\section{Diagnosis \\ Imaging tests}

Compression ultrasonography is now the imaging test of choice to diagnose DVT. Lack of compressibility of a venous segment is the diagnostic criterion used, but the addition of Doppler (in- cluding colour flow) can be useful to accurately identify vessels and to confirm the compressibility of a particular segment.

In many centres, ultrasound testing is limited to the proximal veins (from the common femoral vein caudally to the region of the calf veins where they join the popliteal vein), for which the sensitivity is $97 \%$. For DVT in the calf veins the sensitivity is only $73 \% .{ }^{13}$ Since the distal calf is not scanned, it has been demonstrated that the ultrasound should be repeated I week later (serial testing) if the result is negative to detect DVT extending into the proximal veins. ${ }^{9}$ However, in symptomatic patients, only $20 \%$ of thrombi detected are isolated to the calf, and only $20 \%-30 \%$ of these thrombi will eventually extend to the proximal venous system. (See Fig. I for the anatomy of the deep veins of the leg.) Therefore, routine serial testing is inefficient and inconvenient. Indeed, studies using the serial testing approach have shown that only $\mathrm{r} \%-2 \%$ of patients who have a negative initial ultrasound result will be confirmed to have proximal DVT upon serial testing. ${ }^{13,14}$ As a result, serial testing is not cost-effective. ${ }^{15,16}$

\section{Clinical prediction rules}

Although none of the symptoms or signs of DVT is diagnostic in isolation, it has been well established that a clinical prediction rule that takes into account signs, symptoms and risk factors can be accurately applied to categorize patients as having low, moderate or high probability of DVT (Table I). Alternatively, the same rule can be used to categorize cases as "DVT likely" or "DVT unlikely." ${ }^{17}$ Over I4 studies have demonstrated the reproducibility of this model. ${ }^{18}$ Patients who are found to be at low pretest probability can have DVT safely excluded on the basis of a single negative ultrasound result. ${ }^{10}$ Thus, serial ultrasound testing can be avoided in this subgroup of patients. The incorporation of plasma D-dimer testing into diagnostic algorithms can identify patients who do not require ultrasonography. ${ }^{17}$

\section{D-dimer testing}

D-dimer is a degradation product of a cross-linked fibrin blood clot. Levels of D-dimer are typically elevated in patients with acute venous thromboembolism, as well as in patients with a variety of nonthrombotic conditions (e.g., recent major surgery, hemorrhage, trauma, pregnancy or cancer). ${ }^{19} \mathrm{D}$ dimer assays are, in general, sensitive but nonspecific markers of DVT. The value of the D-dimer assay resides with a negative test result that suggests a lower likelihood of DVT, thus making it a good "rule out" test with the appropriate pretest 
probability. If applied properly, incorporation of D-dimer testing into diagnostic algorithms simplifies the management of a patient presenting with suspected DVT.

\section{Algorithm approach to DVT diagnosis}

Patients with symptoms compatible with DVT should initially have a determination of pretest probability using an established prediction model (Table I) ${ }^{17}$ It is important that a history and physical exam be done first. The model should be applied only if DVT remains a diagnostic possibility. After the clinical pretest probability is determined, a $\mathrm{D}$-dimer test should be performed. In our centre, a score of less than I (unlikely DVT) by our current model, which incorporates previously documented DVT as a new variable, is sufficient to exclude DVT in patients with a negative moderately sensitive D-dimer level without ultrasound imaging. ${ }^{17}$ No D-dimer assay should be used to exclude DVT in patients who have high pretest probability. Clinical assessment and D-dimer testing have the further advantage of enabling the management of patients with suspected DVT who present at times when radiographic imaging is not routinely available. Patients in whom there is a moderate or high clinical suspicion of
DVT may receive an injection of low-molecular-weight (LMW) heparin in doses designed to treat acute DVT. Diagnostic imaging can then be arranged on a more elective basis the following day. Since LMW heparin therapy is safe and effective for patients with proven DVT, it provides adequate protection for patients with suspected DVT. ${ }^{20,21}$ For patients whose risk of DVT is low (as determined either by means of a clinical diagnostic model or a sensitive $\mathrm{D}$-dimer test), diagnostic imaging may be delayed for I2-24 hours without the need for anticoagulant coverage. $.^{10} \mathrm{Ac}-$ cepted algorithms using our prediction model are outlined in Fig. 2. The clinical prediction rule was developed and validated predominantly in studies involving outpatients. Pregnant women were not included in these studies. Furthermore, the utility of the D-dimer test in patients admitted to hospital who often have other comorbidities (e.g., infection, postoperative symptoms) is lower since the D-dimer assay rarely yields negative results. Finally, if DVT is not a diagnostic possibility, a Ddimer test should not be done, because a positive result may redirect a clinician away from investigating the true cause of the leg symptoms toward unnecessarily investigating for DVT.

The ideal strategy for diagnosing DVT in patients who have previously had DVT in the symptomatic leg is still a subject of debate. However, results of a randomized trial demonstrated the safety of combining clinical probability, D-dimer and ultrasound imaging in these patients. ${ }^{17}$ The biggest concern with this patient population is false-positive ultrasound results. It is helpful to recognize that acute DVT is usually occlusive, not echogenic, and it tends to be continuous. If the ultrasound reveals thrombosis that is echogenic, nonocclusive or discontinuous, then chronic DVT should be considered. Serial testing or venography can help to clarify the issue. Previous ultrasound results are helpful for comparison, when available. An increase in clot diameter by $4 \mathrm{~mm}$ suggests recurrence, as does extension. ${ }^{22}$

Most diagnostic and treatment studies of DVT have excluded pregnant women, and therefore it is difficult to formulate evidence-based recommendations for this population. Although serial impedance plethysmography has been demonstrated to safely rule out DVT, ${ }^{23}$ it is not widely used. Results of a small pilot study suggest that a strategy involving serial compression ultrasonography combined with a moderately sensitive Ddimer assay is effective in excluding DVT in pregnant women. ${ }^{24} \mathrm{D}$-dimer levels are often positive in the later stages of pregnancy, ${ }^{25,26}$ lowering the utility of this test to rule out DVT. Research to develop algorithms to diagnose DVT in pregnant women is ongoing. 


\section{Treatment}

The goal of the therapy for lower-extremity DVT is to prevent the extension of thrombus and pulmonary embolism in the short-term and to prevent recurrent events in the long-term. Based on extensive research evaluating the risk of recurrent DVT, guidelines have been established for the duration of anticoagulation therapy. LMW heparin therapy has changed the landscape of treatment of DVT by enabling home treatment and by providing an alternative long-term anticoagulant for people for whom warfarin is less effective or contraindicated. The following pertains to treatment of proximal lowerextremity DVT, since there is little evidence to formulate recommendations for isolated DVT in calf veins.

\section{Initial choice of anticoagulation}

Initial therapy must involve therapeutic doses of either unfractionated heparin or LMW heparin. Initial treatment with oral anticoagulant therapy alone is unacceptable. ${ }^{27}$ The ease of administration and efficacy of LMW heparin make this the preferred anticoagulant, whether given on an outpatient or an inpatient basis. In a meta-analysis comparing the effectiveness of LMW heparin at a fixed dose with unfractionated heparin at an adjusted dose, significantly fewer deaths, major hemorrhage and recurrent venous thromboembolism occurred with the LMW heparin. ${ }^{28}$ Thus, the current standard of care is to administer weight-adjusted LMW heparin once daily for 5-7 days as initial treatment. It remains unknown whether it is better to administer LMW heparin once or twice daily. The results of a meta-analysis suggested that hemorrhage and recurrent venous thromboembolism were less likely to occur with twice daily dosing, but the $95 \%$ confidence interval on the odds ratio crossed I.o. ${ }^{29}$ Since LMW heparin is predominantly renally excreted, unfractionated heparin should be used in patients with significant renal dysfunction. A newer agent is the synthetic pentasaccharide fondaparinux, which is at least as effective and safe as LMW heparin in the treatment of DVT. ${ }^{30}$ Fondaparinux can be considered as an alternative agent for the treatment of DVT with the added benefit that, to date, heparin-induced thrombocytopenia has not been reported with this agent. Unfortunately, the therapeutic dose formulation of fondaparinux (7.5 mg subcutaneously for most patients) currently is not available in Canada.

Early studies evaluating the outpatient treatment of patients with DVT determined that this practice is safe and effective in selected patients. ${ }^{31,32}$ Subsequently, it was demonstrated that a wide spectrum of patients (over $80 \%$ of those with DVT at our institution) could be treated as outpatients. ${ }^{33}$ This practice leads to an improved quality of life for the patients and cost savings for the health care system..$^{34}$ Situations that may necessitate inpatient treatment include comorbidities requiring hospital management, renal failure, high bleeding risk (e.g., recent gastrointestinal hemorrhage), extensive DVT that leads to phlegmasia cerulea dolens, necessity for parenteral narcotics for pain control and an inability to have injections administered at home.

\section{Long-term treatment}

For the majority of patients with DVT, oral therapy with vitamin $\mathrm{K}$ antagonists (e.g., warfarin) is very effective for long-term prevention of recurrent thrombosis. ${ }^{35}$ Although the initial treatment of DVT is similar for most patients, the duration of long-term treatment varies depending on the perceived risk of recurrent DVT. The risk can be classified into the following 5 categories:

- First proximal DVT occurs in the context of a transient risk factor (e.g., surgery or trauma). In this situation, the risk of recurrence is very low and a limited duration of therapy ( 3 months) is adequate. ${ }^{36,37}$

- First DVT occurs in the context of active malignant disease, which is an ongoing risk factor. Patients with malignant disease have a higher incidence of recurrent thrombosis and bleeding complications while receiving oral anticoagulation therapy following a first thrombotic event. ${ }^{38,39}$ This is likely due to the prothrombotic state associated with cancer and to the difficulty of managing oral anticoagulant therapy with concomitant drugs, erratic oral intake and liver dysfunction. Researchers with the CLOT trial ${ }^{40}$ have shown that longterm anticoagulation therapy with LMW heparin is more effective than warfarin at preventing recurrent venous thrombosis without a statistically significant increase in bleeding risk. It is our practice to give all patients who have active malignant disease LMW heparin for at least 6 months if there is adequate renal function. Not only will it lead to lower risks of recurrent thrombosis in many patients, but it facilitates the management of patients who need to undergo multiple procedures (e.g., biopsy, line insertion) and who have periodic thrombocytopenia due to chemotherapy. Since the risk of recurrence is high (2-3 fold higher among patients with cancer than among those without cancer) ${ }^{41}$ treatment with

Table 1: Clinical model for predicting pretest probability of deep-vein thrombosis (DVT)*

\begin{tabular}{lc}
\hline Clinical characteristic $†$ & Score \\
\hline $\begin{array}{l}\text { Active cancer (treatment ongoing, administered } \\
\text { within previous } 6 \text { mo or palliative) }\end{array}$ & 1 \\
$\begin{array}{l}\text { Paralysis, paresis or recent plaster immobilization } \\
\text { of the lower extremities }\end{array}$ & 1 \\
$\begin{array}{l}\text { Recently bedridden > } 3 \text { d or major surgery within } \\
\text { previous } 12 \text { wk requiring general or regional } \\
\text { anesthesia }\end{array}$ & 1 \\
$\begin{array}{l}\text { Localized tenderness along the distribution of } \\
\text { the deep venous system }\end{array}$ & 1 \\
$\begin{array}{l}\text { Swelling of entire leg } \\
\text { Calf swelling > } 3 \text { cm larger than asymptomatic side } \\
\text { (measured } 10 \text { cm below tibial tuberosity) }\end{array}$ & 1 \\
$\begin{array}{l}\text { Pitting edema confined to the symptomatic leg } \\
\text { Collateral superficial veins (nonvaricose) }\end{array}$ & 1 \\
\hline $\begin{array}{l}\text { Previously documented DVT } \\
\text { Alternative diagnosis at least as likely as DVT }\end{array}$ & 1 \\
\hline "A score of 2 or higher indicates that the probability of DVT is “likely"; a score \\
of less than 2 indicates that the probability is "unlikely." \\
†n patients who have symptoms in both legs, the more symptomatic leg is used.
\end{tabular}


anticoagulation drugs is recommended as long as the cancer is felt to be active. We wait 6 months after cure or complete remission before stopping therapy.

- First DVT occurs in the context of a thrombophilic defect. These defects include factor $\mathrm{V}$ Leiden, prothrombin gene mutation, deficiencies in protein $\mathrm{C}$, protein $\mathrm{S}$ and antithrombin, increased factor VIII levels, hyperhomocysteinemia and elevated antiphospholipid antibody levels. Many of these defects are associated with an increased risk of a first DVT. Patients with persistently elevated antiphospholipid antibody levels determined by either ELISA or clotting assays have a 2-fold higher relative risk of recurrence within 4 years after stopping anticoagulation therapy for a first DVT than those without this thrombophilia. ${ }^{37}$ It has been reported that patients with an elevated factor VIII level (above the goth percentile of normal) have a 2-year risk of recurrence of $37 \%$ after stopping anticoagulant agents, compared with $5 \%$ among those with normal levels. ${ }^{43}$ However, this study included lower risk calf vein thrombosis, which may explain the wide difference. In general, the risk of recurrence after a first idiopathic DVT is not influenced by the presence or absence of most thrombophilic defects ${ }^{41}$ and, with the exception of patients with elevated antiphospholipid antibody levels and combined or homozygous genetic defects, ${ }^{43}$ we do not routinely recommend prolonged anticoagulation therapy in these populations after a first idiopathic DVT.
- Recurrent DVT. After a second recurrence of DVT, the risk of further thromboembolic events following the discontinuation of anticoagulation therapy is felt to be excessive if only 6 months of oral anticoagulation therapy is administered. ${ }^{44}$ Therefore, we generally recommend that anticoagulation therapy be continued in this situation. During yearly visits bleeding risk can be assessed, which will enable a risk-benefit evaluation to determine if anticoagulation therapy should continue. However, no study has looked at the risk of recurrent DVT if both events occurred during a transient risk period. In this situation, a shorter duration of anticoagulation therapy may be adequate (3-6 months), but other factors may influence this decision.

- First DVT occurs in the absence of temporary or identifiable ongoing risk factors for thrombosis (idiopathic). Six months is considered a minimum duration for anticoagulation therapy in these patients, while continuing for longer is effective in preventing thrombosis. However, the risk of recurrent venous thromboembolism in the first year after stopping anticoagulation therapy is about 10\%, regardless of when the therapy is stopped after 6 months. ${ }^{45}$ When considering prolonging anticoagulation therapy after 6 months, the risks of bleeding with long-term anticoagulation therapy must be individualized and weighed against the potential benefits of preventing recurrence of thrombosis.

In addition to the thrombophilic defects described previ-

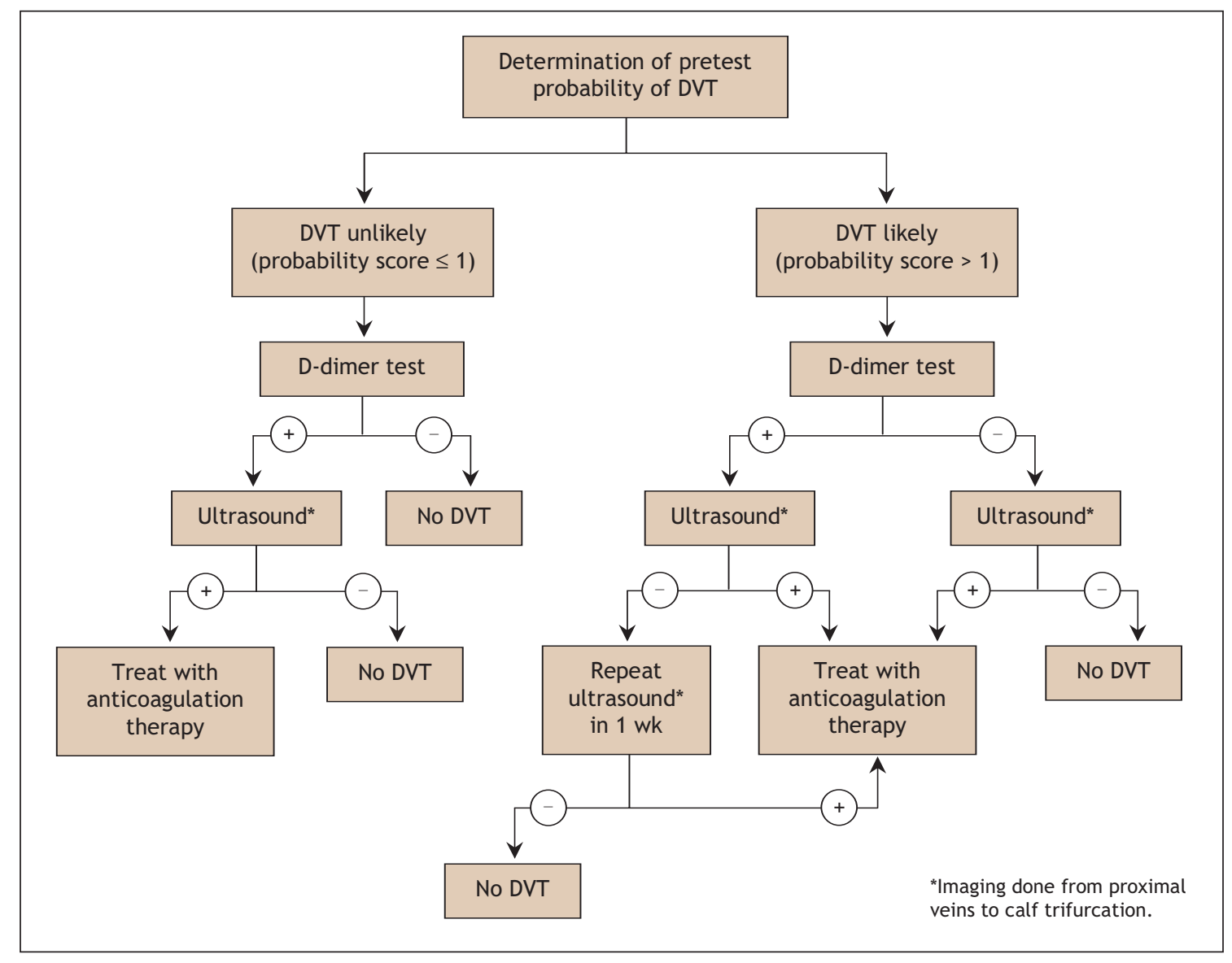

Fig. 2: Diagnostic algorithm using D-dimer testing and ultrasound imaging in patients with suspected DVT. 
ously, 2 factors have been shown to increase the risk of recurrence after stopping anticoagulation therapy. Residual thrombosis (seen on a follow-up ultrasound scan 3 months after an initial event) increases the risk of recurrence (odds ratio 2.4) ${ }^{46}$ One-third of the recurrences occur in the initially unaffected leg, which suggests that residual DVT is a marker of systemic hypercoaguability. In one study, elevated D-dimer levels I month after stopping anticoagulation therapy were associated with an elevated risk of recurrent thrombosis in all but cancer-related thrombosis. ${ }^{47}$ However, it is unclear how to incorporate these factors into clinical decision-making. In an attempt to provide clinical guidelines, our Venous Thromboembolism Clinical Trials group (VECTOR) is conducting a study designed to create a decision rule on recurrence risk.

\section{Intensity of anticoagulation therapy}

The standard intensity of oral anticoagulation therapy is an international normalized ratio (INR) of 2 to 3 . In patients who have antiphospholipid antibody-related thrombosis, it has long been felt that higher intensity anticoagulation therapy is needed to prevent recurrence. ${ }^{48}$ However, results of 2 randomized controlled trials showed that standard anticoagulation therapy is as effective as high-intensity treatment, even in this subgroup of patients. ${ }^{49,50}$ Therefore, high-intensity anticoagulation therapy is not recommended in any patient with DVT. Maintaining good INR control will decrease the risk of postphlebitic syndrome. ${ }^{51}$ There has also been debate on the usefulness of long-term low-intensity anticoagulation therapy (INR I.5-I.9) to prevent recurrent thrombosis while reducing the risk of bleeding. A large randomized trial has shown that low-intensity anticoagulation therapy is less effective than standard anticoagulation therapy at preventing recurrent thrombosis and does not lower the risk of bleeding. ${ }^{52}$ Therefore, low-intensity therapy is not recommended.

\section{Upper-extremity DVTs}

Upper-extremity DVTs can be subdivided into catheter- and noncatheter-related thrombosis. There is a risk of pulmonary embolism with this condition, and therefore treatment with anticoagulation therapy is generally recommended. Initial treatment with thrombolytic therapy for acute upper-extremity DVT has been used with some success, but no randomized controlled trials comparing thrombolytic therapy with anticoagulation therapy alone have been performed. A more detailed discussion of upper-extremity DVT is beyond the scope of this article, and we would refer the reader to a review addressing this topic. ${ }^{53}$

\section{Special patient populations}

The treatment of DVT during pregnancy deserves special mention, since oral anticoagulation therapy is generally avoided during pregnancy because of the teratogenic effects in the first trimester and the risk of fetal intracranial bleeding in the third trimester. LMW heparin is the treatment of choice for DVT during pregnancy. If acute DVT occurs near term, interrupting anticoagulation therapy may be hazardous because of the risk of pulmonary embolism. In this situation, placement of a retrievable inferior vena cava filter must be considered. However, there is no consensus as to what the appropriate dose should be and whether anti-Xa levels need to be monitored. This topic is well discussed in a recent review. ${ }^{54}$

For obese patients with DVT, results of a registry study suggest that they have similar outcomes as nonobese patients with DVT. ${ }^{55}$ The dose of LMW heparin does not need to be capped, and monitoring is not required, except perhaps in people who are morbidly obese, since fewer data are available for these patients. ${ }^{56,57}$

\section{Other interventions}

Although anticoagulation therapy is the mainstay of treatment of DVT, thrombolysis and placement of an inferior vena cava filter are 2 interventions that deserve mention. The addition of systemic thrombolysis to standard anticoagulation therapy leads to earlier patency of an occluded vein; however, it does not affect the rate of pulmonary embolism. There is a definite increase in the risk of major hemorrhage, including intracranial hemorrhage, with thrombolysis. Catheter-directed thrombolysis has also been associated with increased risk of bleeding complications. It is unclear whether the earlier recanalization seen with thrombolysis translates into lower rates of postthrombotic syndrome over the long term. ${ }^{58,59}$ Thrombolysis is not generally recommended except in the case of massive DVT, which leads to phlegmasia cerulean dolens and threatened limb loss.

Placement of an inferior vena cava filter in addition to anticoagulation therapy has not been found to prolong survival among patients with DVT. While preventing pulmonary embolism, insertion of a filter increases the risk of recurrent DVT. ${ }^{60,61} \mathrm{~A}$ retrievable filter is indicated when there is a contraindication to anticoagulation therapy (recent hemorrhage, impending surgery) in patients with newly diagnosed proximal DVT. It remains to be determined if a retrievable filter in patients at higher risk of death (e.g., limited cardiopulmonary reserve) will lead to a reduction in pulmonary embolism-related death.

Postphlebitic syndrome is a frequent complication of DVT and a major public health issue that has been underresearched. It is unclear who is at highest risk and how best to prevent and treat this complication. Some data suggest a potential benefit from the use of graduated compression stockings, and our VECTOR group is currently investigating this issue in a randomized trial. Postphlebitic syndrome is well reviewed in a recent publication. ${ }^{7}$

This article has been peer reviewed.

From the Departments of Medicine (Scarvelis, Wells) and of Community Medicine and Epidemiology (Wells), the Division of Hematology (Scarvelis, Wells), and the Ottawa Health Research Institute (Scarvelis, Wells), University of Ottawa, Ottawa, Ont.

Competing interests: None declared for Dimitrios Scarvelis. Philip Wells has received honoraria for speaker fees from Leo Pharma, Sanofi-Aventis and AstraZeneca, and sits on a steering committee for a study of a new anticoagulant sponsored by Bayer.

Contributors: Both authors contributed substantially to the manuscript, including drafting the article and revising it critically for important intellectual content, and approved the submitted version. 


\section{REFERENCES}

I. White RH. The epidemiology of venous thromboembolism. Circulation 2003; ro7(Suppl I): I4-8.

2. Silverstein MD, Heit JA, Mohr DN, et al. Trends in the incidence of deep vein thrombosis and pulmonary embolism: a 25-year population-based study. Arch Intern Med I998; I58:585-93.

3. Hirsh J, Bates SM. Prognosis in acute pulmonary embolism. Lancet 1999;353: I375-6.

4. Prandoni P, Lensing AWA, Prins M. Long-term outcomes after deep venous thrombosis of the lower extremities. Vasc Med i $998 ; 3: 57-60$.

5. Fedullo PF, Auger WR, Channick RN, et al. Chronic thromboembolic pulmonary hypertension. Clin Chest Med 200I;22:56I-8I.

6. Pengo V, Lensing AW, Prins MH, et al. Incidence of chronic thromboembolic pulmonary hypertension after pulmonary embolism. N Engl J Med 2004;350:2257-64.

7. Kahn SR, Ginsberg JS. Relationship between deep venous thrombosis and the postthrombotic syndrome. Arch Intern Med 2004;164:17-26.

8. Wells PS, Anderson DR. Diagnosis of deep-vein thrombosis in the year 2000. Curr Opin Pulm Med 2000;6:309-I3.

9. Cogo A, Lensing AWA, Koopman MMW, et al. Compression ultrasonography for diagnostic management of patients with clinically suspected deep vein thrombosis: prospective cohort study. BMJ I998;316:17-20.

Io. Wells PS, Anderson DR, Bormanis J, et al. Value of assessment of pretest probability of deep-vein thrombosis in clinical management. Lancet 1997;350:1795-8.

II. Bates SM, Kearon C, Crowther MA, et al. A diagnostic strategy involving a quantitative latex D-dimer assay reliably excludes deep venous thrombosis. Ann Intern Med 2003;138:787-94.

I2. Heijboer H, Buller HR, Lensing AWA, et al. A comparison of real-time compression ultrasonography with impedance plethysmography for the diagnosis of deepvein thrombosis in symptomatic outpatients. N Engl J Med I993;329:1365-9.

13. Kearon C, Julian JA, Newman TE, et al. Noninvasive diagnosis of deep vein thrombosis. Ann Intern Med I998;128:663-77.

I4. Wells PS, Lensing AWA, Davidson BL, et al. Accuracy of ultrasound for the diagnosis of deep venous thrombosis in asymptomatic patients after orthopedic surgery. A meta-analysis. Ann Intern Med I995;I22:47-53.

I5. Perone N, Bounameaux H, Perrier A. Comparison of four strategies for diagnosing deep vein thrombosis: a cost-effectiveness analysis. Am J Med 2000;110:33-40.

I6. Hillner BE, Philbrick JT, Becker DM. Optimal management of suspected lower-extremity deep vein thrombosis. An evaluation with cost assessment of 24 management strategies. Arch Intern Med I992;152:165-75.

17. Wells PS, Anderson DR, Rodger M, et al. Evaluation of D-Dimer in the diagnosis of suspected deep-vein thrombosis. N Engl J Med 2003;349:1227-35.

I8. Wells PS, Owen C, Doucette S, et al. Does this patient have deep vein thrombosis? JAMA 2006;295:199-207.

I9. Kelly J, Rudd A, Lewis RR, et al. Plasma D-dimers in the diagnosis of venous thromboembolism. Arch Intern Med 2002;162:747-56.

20. Bauld DL, Kovacs MJ. Dalteparin in emergency patients to prevent admission prior to investigation for venous thromboembolism. Am J Emerg Med i999;17:II-4.

2I. Anderson DR, Kovacs MJ, Kovacs G, et al. Combined use of clinical assessment and D-dimer to improve the management of patients presenting to the emergency department with suspected deep-vein thrombosis (the EDITED Study). J Thromb Haemost 2003;1:645-5I.

22. Heijboer H, Jongbloets LM, Buller HR, et al. Clinical utility of real-time compression ultrasonography for diagnostic management of patients with recurrent venous thrombosis. Acta Rad i992;33:297-300.

23. Hull RD, Raskob GE, Carter CJ. Serial impedance plethysmography in pregnant patients with clinically suspected deep-vein thrombosis. Clinical validity of negative findings. Ann Intern Med I990;II 2:663-7.

24. Chan WS, Chunilal SD, Lee AY, et al. Diagnosis of deep vein thrombosis during pregnancy: a pilot study evaluating the role of D-dimer and compression leg ultrasound during pregnancy. Blood 2002;100:275a.

25. Epiney $M$, Boehlen $F$, Boulvain $M$, et al. D-dimer levels during delivery and the postpartum. J Thromb Haemost 2005;3:268-7I.

26. Eichinger S. D-dimer testing in pregnancy. Pathophysiol Haemost Thromb 2003; 33:327-9.

27. Brandjes DPM, Heijboer H, Buller HR, et al. Acenocoumarol and heparin compared with acenocoumarol alone in the initial treatment of proximal-vein thrombosis. N Engl J Med I992;327:1485-9.

28. Van Dongen CJ, van den Belt AG, Prins MH, et al. Fixed dose subcutaneous low molecular weight heparins versus adjusted dose unfractionated heparin for venous thromboembolism [review]. Cochrane Database Syst Rev. 2004 Oct I8;(4):CDooIIoo.

29. Van Dongen CJ, Mac Gillavry MR, Prins MH. Once versus twice daily LMWH for the initial treatment of venous thromboembolism [review]. Cochrane Database Syst Rev. 2003;(I):CDo03074.

30. Matisse Investigators. Fondaparinux or enoxaparin for the initial treatment of symptomatic deep vein thrombosis. A randomized trial. Ann Intern Med 2004;140:867-73.

3I. Levine M, Gent M, Hirsh J, et al. A comparison of low molecular weight heparin administered primarily at home with unfractionated heparin administered in the hospital for proximal deep vein thrombosis. N Engl J Med I996;334:677-8I.

32. Koopman MMW, Prandoni P, Piovella F, et al. Treatment of venous thrombosis with intravenous unfractionated heparin administered in the hospital as compared with subcutaneous low molecular weight heparin administered at home. $\mathrm{NEngl} \mathrm{J}$ Med I996;334:682-7.
33. Wells PS, Kovacs MJ, Bormanis J, et al. Expanding eligibility for outpatient treatment of deep venous thrombosis and pulmonary embolism with low molecular weight heparin: a comparison of patient self-injection with homecare injection. $N$ Engl J Med 1998;158:1809-12.

34. Rodger MA, Gagné-Rodger C, Howley HE, et al. The outpatient treatment of deep vein thrombosis delivers cost savings to patients and their families compared to inpatient therapy. Thromb Res 2003;112:13-8.

35. Hull R, Hirsh J, Jay R, et al. Different intensities or oral anticoagulant therapy in the treatment of proximal-vein thrombosis. N Engl J Med I982;307:I676-8I.

36. Levine MN, Hirsh J, Gent M, et al. Optimal duration of oral anticoagulation therapy: a randomized trial comparing four weeks with three months of Warfarin in patients with proximal deep vein thrombosis. Thromb Haemost 1995;74:606-II.

37. Schulman S, Rhedin AS, Lindmarker P, et al. A comparison of six weeks with six months of oral anticoagulant therapy after a first episode of venous thromboembolism: Duration of Anticoagulation Trial Study Group. N Engl J Med I995;332:I66I-5.

38. Hansson PO, Sorbo J, Eriksson H. Recurrent venous thromboembolism after deep vein thrombosis: incidence and risk factors. Arch Intern Med 2000;160:769-74.

39. Prandoni P, Lensing A, Piccioli A, et al. Recurrent venous thromboembolism and bleeding complications during anticoagulation treatment in patients with cancer and venous thrombosis. Blood 2002;100:3484-8.

40. Lee AY, Levine MN, Baker RI, et al. Low-molecular-weight heparin versus coumadin for prevention of recurrent venous thromboembolism in patients with cancer. N Engl J Med 2003;349:I46-53.

4I. Baglin T, Luddington R, Brown $\mathrm{K}$, et al. Incidence of recurrent venous thromboembolism in relation to clinical and thrombophilic risk factors: prospective cohort study. Lancet 2003;362:523-6.

42. Kyrle PA, Minar E, Hirschl M, et al. High plasma factor VIII and the risk of recurrent venous thromboembolism. N Engl J Med 2000;343:457-62.

43. De Stefano V, Martinelli I, Mannucci PM, et al. The risk of recurrent deep venous thrombosis among heterozygous carriers of both factor V Leiden and the G202IoA prothrombin mutation. N Engl J Med I999;34I:80I-6.

44. Schulman S, Granqvist S, Holmstrom M, et al. The duration of anticoagulation after a second episode of venous thromboembolism. N Engl J Med i997;336:393-8.

45. Ost D, Tepper J, Mihara H. Duration of anticoagulation following venous thromboembolism: A meta-analysis. JAMA 2005;294:706-I5

46. Prandoni P, Lensing AWA, Prins $M H$, et al. Residual thrombosis as a predictive factor of recurrent venous thrombosis. Ann Intern Med 2002;137:955-60.

47. Cushman M, Folsom AR, Wang L, et al. Fibrin fragment D-dimer and the risk of future venous thrombosis. Blood 2003; I0I:1243-8.

48. Khamashta MA, Cuadrado MJ, Mujic F, et al. The management of thrombosis in the antiphospholipid-antibody syndrome. N Engl J Med I995;332:993-7.

49. Crowther MA, Ginsberg JS, Julian J, et al. A comparison of two intensities of warfarin for the prevention of recurrent thrombosis in patients with the antiphospholipid antibody syndrome. NEngl J Med 2003;349:II33-8.

50. Finazzi G, Marchioli R, Brancaccio V, et al. A randomized clinical trial of highintensity warfarin vs. conventional antithrombotic therapy for the prevention of recurrent thrombosis in patients with the antiphospholipid syndrome (WAPS). $J$ Thromb Haemost 2005;3:848-53.

5I. Van Dongen CJ, Prandoni P, Frulla M, et al. Relation between quality of anticoagulant treatment and the development of the postthrombotic syndrome. J Thromb Haemost 2005;3:939-42.

52. Kearon C, Ginsberg JS, Kovacs MJ, et al. Comparison of low-intensity warfarin therapy with conventional-intensity warfarin therapy for long-term prevention of recurrent venous thromboembolism. NEngl J Med 2003;349:63I-9.

53. Bernardi E, Piccioli A, Marchiori A, et al. Upper extremity deep vein thrombosis: risk factors, diagnosis, and management. Semin Vasc Med 200I; I:I05-Io.

54. Greer I, Hunt BJ. Low molecular weight heparin in pregnancy: current issues. $\mathrm{Br}$ Haematol 2005;128:593-6or.

55. Barba R, Marco J, Martin-Alvarez H, et al. The influence of extreme body weight on clinical outcome of patients with venous thromboembolism: findings from a prospective registry (RIETE). J Thromb Haemost 2005;3:856-62.

56. Al Yaseen E, Wells PS, Anderson J, et al. The safety of dosing dalteparin based on actual body weight for the treatment of acute venous thromboembolism in obese patients. J Thromb Haemost 2005;3:100-2.

57. Wilson SJ, Wilbur K, Burton E, et al. Effect of patient weight on the anticoagulant response to adjusted therapeutic dosage of low-molecular-weight heparin for the treatment of venous thromboembolism. Haemostasis 2001;31:42-8.

58. Wells PS, Forster AJ. Thrombolysis in deep vein thrombosis: Is there still an indication? Thromb Haemost 2001;86:499-508.

59. Forster AJ, Wells PS. Tissue plasminogen activator for the treatment of deep venous thrombosis of the lower extremity: a systematic review. Chest 200I;II9:572-9.

6o. Decousus H, Leizorovicz A, Parent F, et al. A clinical trial of vena cava filters in the prevention of pulmonary embolism in patients with proximal deep-vein thrombosis. N Engl J Med I $998 ; 338: 409-\mathrm{I} 6$.

6r. Decousus H, Barral F-G, Buchmuller-Cordier A, et al. Eight-year follow-up of patients with permanent vena cava filters in the prevention of pulmonary embolism the PREPIC (Prévention du Risque d'Embolie Pulmonaire par Interruption Cave) randomized study. Circulation 2005;112:416-22.

Correspondence to: Dr. Dimitrios Scarvelis, Division of Hematology, The Ottawa Hospital, Rm. 462, 737 Parkdale Ave., Ottawa ON KIY IJ8; fax6I376I-4840; dscarvelis@ottawahospital.on.ca 